\title{
Bacterial Probiotics their Importances and Limitations: A Review
}

\author{
Ayichew $\mathrm{T}^{* 1}$, Belete $\mathrm{A}^{1}$, Alebachew $\mathrm{T}^{1}$, Tsehaye $\mathrm{H}^{1}$, Berhanu $\mathrm{H}^{2}$ and Minwuyelet $\mathrm{A}^{3}$ \\ ${ }^{1}$ Wolaita Sodo University School of Veterinary Medicine, Wolaita, Ethiopia \\ ${ }^{2}$ Alage Agricultural Research Institute, Ethiopia \\ ${ }^{3}$ Bure College of Agriculture, Branch of Debre Markos University Bure, Ethiopia
}

*Corresponding author: Ayichew T, Wolaita Sodo University School of Veterinary Medicine, Wolaita, Ethiopia, E-mail: tayichew25@gmail.com

Citation: Ayichew T, Belete A, Alebachew T, Tsehaye H, Berhanu H, et al. (2017) Bacterial Probiotics their Importances and Limitations: a Review. J Nutr Health Sci 4(2): 202. doi: 10.15744/2393-9060.4.202

Received Date: March 31, 2017 Accepted Date: May 29, 2017 Published Date: May 31, 2017

\begin{abstract}
Every animal hosts large number of microorganisms most of which affect its body positively. Such positive effect is induced by probiotics, live microbial food ingredients that alter the enteric micro flora and have a beneficial effect on the health of an animal. There are many different varieties of friendly bacteria that exist naturally in foods or drinks, or can be added to foods or beverages to enhance health and wellbeing of animals. Thermal treatment and aseptic production of food strongly reduces the amount of microorganisms taken up. This alters the microbial composition in our digestive system. The historical association of probiotics with fermented dairy products, still true today, stems from these early observations. Most conventional forms of drug therapy suppress or modify the host immune-inflammatory response and neglect the other contributor to disease pathogenesis, the environmental micro- flora. This paper provides an overview of the current status of the proposed bacterial probiotics, their importance and limitation, their mechanism of work, and some safety considerations.
\end{abstract}

Keywords: Bacteria, Importance, Micro flora, Probiotics

\section{Introduction}

The term "probiotics" comes from the Greek words "pro" (in favor) and "biotic" (life). Probiotics are defined as "live microorganisms that may beneficially affect the host upon ingestion by improving the balance of the intestinal micro flora" [1]. The terms direct-fed microbial and probiotics are used interchangeably [2]. More recently definition: "Mono or mixed cultures of live microorganisms which, when applied to animal or man, beneficially affect the host by improving the properties of the indigenous micro flora". This latest definition is more specific in terms of the host and types of microorganisms and not restricted to the intestinal microbial community [3].

At birth, the gastrointestinal (GI) tract of any animal is sterile and it is rapidly colonized by bacteria from the mother and the environment. This colonization by the gut microbiota plays an important role in intestinal tract maturation, digestive physiology and immunology of newborn [4]. Prior to birth, microorganisms are absent from the GI tract, but quickly colonize it during and after birth. Exactly which microbiota develops is dependent on factors such as: the method of delivery and the environment in which birth takes place, the mother's micro biota and the manner of feeding [5].

In the meantime probiotics are applied as feed supplements, pharmaceuticals, dairy products, fruit juices, chocolates and even meat products [6]. Clinical trials have evaluated the use of probiotics in the prevention and treatment of GI diseases caused by pathogenic microorganisms or by disturbances in the normal micro-flora [7]. The conventional use of probiotics to modulate gastrointestinal health such as: improving lactose intolerance, increasing natural resistance to infectious diseases in the gastrointestinal tract, suppressing traveler's diarrhea and reducing bloating, has been well investigated and documented [6]. Probiotics are intended to assist the body's naturally occurring gut microbiota. Studies have documented probiotic effects on a variety of gastrointestinal and extra intestinal disorders, including inflammatory bowel disease (IBD), irritable bowel syndrome (IBS), vaginal infections and immune enhancement [8]. The crucial event in the development of the probiotic approach to animal health was the finding that the newly hatched chicken could be protected against salmonella colonization of the gut by dosing it with a suspension of gut contents prepared from healthy adult chickens [6].

Probiotics have also received special attention by animal nutrition researchers who search for alternatives to the use of traditional growth promoters (antibiotics). The use of probiotics is seen more and more as an alternative to the use of antibiotics in animal production, and many scientific works show the beneficial effects of supplementation with probiotic strains in diets fed to chicken, 
swine, cattle and fish [9]. The objectives of this paper are:

i. To highlight the nutritional importance and health benefits of probiotics.

ii. To describe safety aspect of bacterial probiotics and their limitations.

iii. To review on the bacterial used in probiotics, selection criteria and mechanism of action.

\section{Bacterial Probiotics}

Microorganisms used in probiotics: The composition of the gastro intestinal tract flora varies between individuals and also within the same individual during life. The tract contains both 'friendly' and pathogenic bacteria that exist in a complex symbiosis. Various factors such as diet, climate, ageing, medication (particularly antibiotic consumption), illness, stress and lifestyle can upset this balance leading to diarrhea, mucosal inflammation or other serious illnesses [10].

The crucial event in the development of the probiotic approach to animal health was the finding that the newly hatched chicken could be protected against salmonella colonization of the gut by dosing it with a suspension of gut contents prepared from healthy adult chickens. Microorganisms used in probiotics include those derived from the Lactobacillus, Streptococcus, Enterococcus, Bacillus, Clostridium, Bifidobacterium species and E. coli [11]. Bacterial probiotics have been effective in chickens, pigs and preruminant calves [6].

Species belonging to Bifidobacterium and Lactobacillus are largely used as probiotics [12]. Most of the probiotic bacteria are lactic acid producing bacteria (LAB). It has been shown that lactic acid inhibited the growth of coliforms in the gastro-intestinal tract. Acidic environments are detrimental to many pathogens [3]. The most commonly used probiotic strains include the lactic acid bacteria (LAB) and Gram-positive microbes that have been used for centuries in food production processes (yogurt, cheese, pickles). Members of the LAB such as Lactococcus and Streptococcus are also important components of the endogenous microbiota in the human ileum and jejunum administered to neonates to prevent the colonization of their digestive tract by multidrugresistant pathogens $[13,14]$.

Probiotics Selection Criteria: Probiotics are viable bacteria that beneficially influence the health of the host. Probiotic bacteria selected for commercial use in foods and in therapeutics must retain the characteristics for which they were originally selected [15]. An ideal probiotic should have several potential characteristics such as, non-pathogenic and non-toxic in nature, beneficial to the host animal, high viability, stable on storage and in the field, able to survive in or colonize the gut, and amenable to cultivation on an industrial scale [16]. In addition to this, a probiotic organisms should meet the following: resistance to gastric acidity, resistance to bile and pancreatic enzymes (PE), adherence to intestinal mucosa cells, colonization capacity, keep itself alive for a long time during transportation, storage, so they can effectively colonize the host; production of antimicrobial substances against pathogenic bacteria and absence of translocation [4].

For food applications, probiotics need to survive until they reach the part of the GI tract where they exert their intended effect. For example, to be active in the colon, probiotics must resist salivary enzymes, stomach acid, small intestinal secretions of bile and enzymes as well as the $\mathrm{pH}$ changes and chemical of other foods and beverages they will encounter during their passage along the GI tract. In addition, they need to compete with the resident microbiota. Finally, a selected strain has to fulfill a number of technological requirements, such as cultivability on a large scale, genetic stability and maintaining viability in a food product or supplement [5].

Firstly, microorganism sources have to be selected (for example: digestive tract of health animals or other niches such as flowers, decomposing fruits). Then, the microorganisms which are intended to work with are isolated and identified through selective culture media. Afterwards, a new culture is prepared only with the target colonies for in vivo assessment (pathogen inhibition, target species pathogenicity; resistance to host conditions; among others). If there are no restriction to the use of the target species, experiments with in vivo supplementation at big and small scale are carried out to check if there are real benefits to the host. Finally, the probiotic which presented significant satisfactory results can be commercially produced and used [4].

\begin{tabular}{|c|c|c|c|}
\hline Lactobacillus species & Bifidobacterium & Other LAB & Non-lactics \\
\hline L. acidophilus & B. adolescentis & Enterococcus & Bacillus cereus \\
\hline L. casei & B. animalis & Faecalis & Escherichia coli \\
\hline L. crispatus & B. bifidum & E. faecium & Propionibacterium \\
\hline L. gallinarum & B. breve & Lactococcus lactis & freudenreichii \\
\hline L. gasser & B. infantis & Leuconostoc & \\
\hline L. johnsonii & B. lactis & Mesenteroides & \\
\hline L. paracasei & B. longum & Pediococcus & \\
\hline L. plantarum & \multicolumn{3}{|c|}{ Acidilactici } \\
\hline L. reuteri & Taborolactobacillus & Inulinus Strep. thermophilus \\
\hline L. rhamnosus & Microorganisms applied in probiotic products [3] \\
\hline \multicolumn{4}{|l}{}
\end{tabular}


The strains were able to compete with normal microflora and resistance to bile and acids can exert one or more clinically documented health benefits [17]. Probiotic producers, medical professionals and public health officers consider some form of system to monitor the health outcomes of long-term probiotic administration. Characterization of each strain of microorganism that used in production of probiotics must be on bases of guideline established by a working group conveyed of jointly by the FAO and WHO, in which they considered the safety probiotic products on human health. Improper usage of the probiotic production guideline results in developments of pathogenic microorganisms for both human and animal health [18].

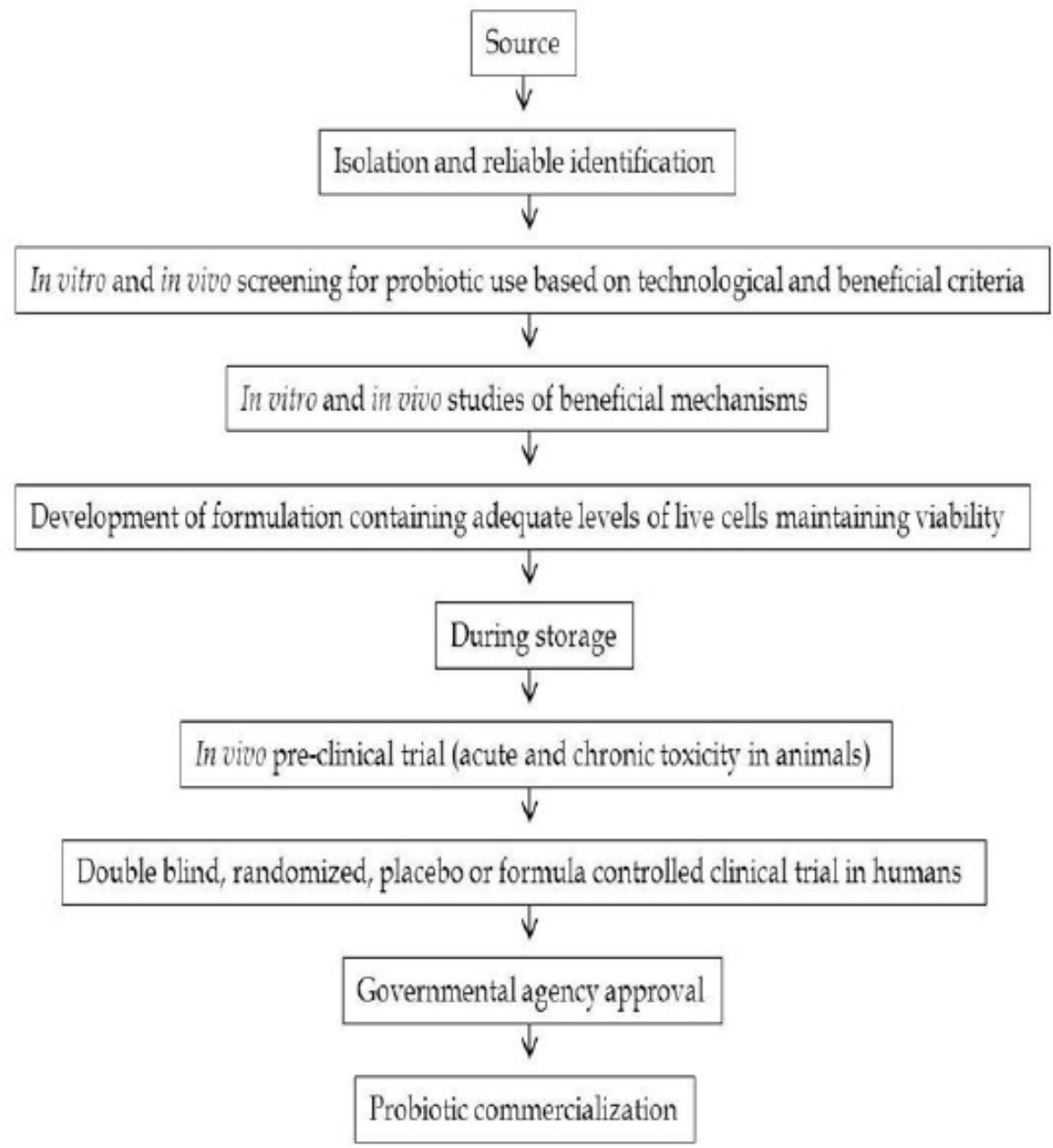

Figure 1: Probiotic selection chart [4]

\section{Mechanism of action of probiotics}

Probiotic bacteria counteract inflammatory processes by stabilizing a healthy microbiota and thus improving the intestine's permeability barrier. In addition to influencing gut microbiota and immune system, other mechanisms of probiotic action have been proposed, such as inhibition of pathogens by competition for nutrients and attachment sites or by production of antimicrobial substances, reduction of cholesterol levels through deconjugation of bile salts or binding of toxins and preventing their absorption [19].

The potential mechanisms by which probiotic agents might exert their protective effect include: antagonism by the production of substances that inhibit or kill the pathogen, competition with the pathogen for adhesion sites or nutritional sources; immunemodulation of the host; and inactivation of microbial toxin (Other mechanisms by which probiotics may exert protection is through a recuperation of mucosal barrier function when disturbed, trapping pathogens on their surface and stimulating mucus production, specific probiotic bacteria have been reported to modulate local and systemic immune responses [20-24]. Although the mechanisms of immune modulation are not fully understood it is known that bacterial components are recognized by the immune system through their interaction with specific Toll-like receptors resulting in the modulation of immune responses [25].

\section{Importance of probiotics}

Many health benefits have been related to human and animal intake of probiotic. Several studies have supplied clinical evidences of the benefits generated by probiotics, as for example in diarrhea treatment, lactose intolerance and irritable bowel syndrome (IBS) [4]. In the meantime probiotics are applied as feed supplements, pharmaceuticals, dairy products, fruit juices, chocolates and even 
meat products [26]. The conventional use of probiotics to modulate gastrointestinal health, such as improving lactose intolerance, increasing natural resistance to infectious diseases in the GI tract, suppressing traveler's diarrhea and reducing bloating, has been well investigated and documented [6]. Clinical trials have evaluated their use in the prevention and treatment of GI diseases caused by pathogenic microorganisms or by disturbances in the normal micro-flora [7]. Recent research on the molecular biology and genomics of Lactobacillus has focused on the interaction with the immune system, anti-cancer potential, and potential as a biotherapeutic agent in cases of antibiotic-associated diarrhea, travelers' diarrhea, pediatric diarrhea, inflammatory bowel disease and irritable bowel syndrome [16].

\section{Nutritional importance}

It is necessary to remember that more than 2000 years ago ancient Greek physician Hippocratus, considered as the father of Western medicine, said," Let food be thy medicine and medicine be thy food." The use of foods for their medical value as for their nutritional benefits is an ancient tradition in China, India, Egypt and Japan [27]. Besides their desired health and clinical properties, probiotics must meet several basic requirements for the development of marketable probiotic products. The most important requirements are that probiotic bacteria survive in sufficient numbers in the product, that their physical and genetic stability during storage of the product be guaranteed, and that all of their properties essential for expressing their health benefits after consumption be maintained during manufacture and storage of the product [28]. Preparation of viable microorganisms that is consumed by humans or other animals with the aim of inducing beneficial effects by qualitatively or quantitatively influencing their gut microflora and/or modifying their immune status [29].

Fermentation is a process in which a microorganism transforms food into other products, usually through the production of lactic acid, ethanol and other metabolic end-products [8]. Probiotic bacteria are sold mainly in fermented foods, and dairy products play a predominant role as carriers of probiotics. These foods are well suited for promoting the positive health image of probiotics for several reasons: fermented foods and dairy products in particular, already have a positive health image; consumers are familiar with the fact that fermented foods contain living microorganisms (bacteria); probiotics used as starter organisms combine the positive images of fermentation and probiotic culture [28].

For centuries it has been considered that a diet based on fermented milk products has a beneficial effect, controlling the development of pathogenic bacteria in the thin intestine, leading to improved overall health, and having as main effect increase life period [30]. Metchnikoff proposed that consumption of fermented milk would "seed" the intestine with harmless lactic-acid bacteria and decrease the intestinal $\mathrm{pH}$ and that this would suppress the growth of pathogenic bacteria [16].

Lactic acid fermentation is the most common method and one of the easiest to experiment with. It is a process where lactic acid bacteria (LAB), mainly the Lactobacillus species, convert sugar into lactic acid, which acts as a preservative. Prior to refrigeration and pasteurization, fermentation allowed food to be stored and preserved for later use, preventing spoilage by the natural defenses of lactic acid producing bacteria [31]. Lactic acid producing bacteria (LAB) are functional classification of nonpathogenic, nontoxigenic, Gram-positive, fermentative bacteria that are associated with the production of lactic acid from carbohydrates, making them useful for food fermentation. Species of Lactobacillus, Lactococcus, and Streptococcus, Thermophilus are included in this group [8].

\section{Public health importance}

Over the last 20 years there has been an increased interest in the role of probiotic bacteria in human health. Some of the major benefits of probiotics are: they reduce or eliminate ailments such as colon irritation, constipation and traveler's diarrhea, inhibition of pathogenic bacteria, synthesis of B vitamins, lowering of blood ammonia levels, improving the absorption of calcium, and improvement of lactose utilization by producing b-galactosidase, when there are enough probiotics in colon [12]. Probiotics are intended to assist the body's naturally occurring gut microbiota. Studies have documented probiotic effects on a variety of GI and extra-intestinal disorders, including inflammatory bowel disease (IBD), irritable bowel syndrome (IBS), vaginal infections and immune enhancement [8].

\section{Improving immune system}

The intake of probiotics has been associated with beneficial effects on the immune system, such as improved disease resistance and reduced risk of allergies [30]. Probiotic in the organism of a healthy animal stimulate non-specific immune response and enhance the system of the immune protection. Probiotic increased intestinal IgA secretion both in sows and piglets and elevated IgG and IgM levels in turkey. The effect of intestinal IgA secretion could be related to a more successful mucosal defense which in turn led to a lower level in systemic IgG production in piglets after weaning [6].

The mechanisms by which epithelial cells are making the difference between probiotic and pathogenic microorganisms appear to be different. Pathogenic bacteria induce a pro-inflammatory response in epithelial cells by activating transcription factor NF-kB. Compared with these bacteria, non-pathogenic species may alleviate to the side of pro-inflammatory response by blocking this factor [32]. The strongest clinical evidence for probiotics is related to their use in improving gut health and stimulating immune function [8]. At present it is believed that there is some relationship between the ability of strain to translocate and the ability to be immunogenic [33]. 
The improvement in the immune system may be by three different ways: enhanced macrophage activity disturbance and enhanced ability to phagocytose microorganism; increased production of antibodies usually of IgG and IgM classes and interferon (a nonspecific antiviral agent) and increased local antibodies at mucosal surfaces such as the gut wall, usually $\operatorname{IgA~[16].~}$

Activate local macrophages to increase antigen presentation to B lymphocytes and increase secretory immunoglobulin A (IgA) production both locally and systemically, modulate cytokine profiles, (Immunologic benefits) and digest food and compete for nutrients with pathogens, alter local $\mathrm{pH}$ to create an unfavorable local environment for pathogens, produce bacteriocins to inhibit pathogens, Scavenge superoxide radicals, stimulate epithelial mucin production, enhance intestinal barrier function (Nonimmunologic benefits) [8]. Consumption of beneficial bacteria such as L. acidophilus and L. casei reinforces intestinal mucous membrane immunity (mucosal immunity) as well as the body's global immunity (systemic immunity) [16]. It has been discovered that conventional animals with a complete gut flora have increased phagocytic activity and immunoglobulin levels compared with germ-free animals [31].

\section{Reducing inflammation and irritable bowel syndrome}

Probiotics act through the induction of regulatory T-cells that suppress inflammation-inducing effectors cells. Probiotics have the potential characteristics to interact with the mucosal immune system that does not arouse an inflammation inducing innate response, and the consequent induction of master inflammatory cytokines [16]. The non-pathogenical strain E. coli showed to be efficient in the Crohn's disease maintenance therapy. This microorganism was able to adhere to intestinal epithelial cells in addition to its inhibitory effect observed against pathogenic strains isolated from patients with the disease [4]. Inflammatory bowel diseases, such as pouchitis and Crohn's disease, as well as irritable bowel syndrome (IBS), may be caused or aggravated by alterations in the gut flora including infection [34].

Some studies support the potential role of probiotics in therapy and prophylaxis and illustrate that combinations of strains may have a role to play in remediation [35]. The intestinal microflora likely plays a critical role in inflammatory conditions in the gut, and potentially probiotics could remediate such conditions through modulation of the microflora [36]. Inflammatory bowel diseases (IBS) are a diseased characterized by abdominal pain, diarrhea, constipation and mucus secretion along with feces [37]. Although many physiopathology factors had been correlated to the cause of this disease, in the last years, researchers have considered feed intolerance and unbalance of intestinal microbiota as the main factors responsible for symptoms of the irritable bowel syndrome. Probiotics are a good alternative for the treatment of this syndrome [4].

The effects of immune system are promising. However, the mechanism is not well understood. Human studies have shown that probiotic bacteria can have positive effects on the immune system of their hosts. Probiotics affect the immune system in different ways such as producing cytokines, stimulating macrophages, increasing secretary IgA concentrations [38].

\section{Probiotics and management of diarrhea}

Bacterial agents are responsible for approximately $10 \%$ of diarrheal illnesses in industrialized countries. Viruses are more important in infants and cause community food and waterborne gastroenteritis in all age-Groups [10].

One of the main applications of probiotic microorganisms is at preventing or in the treatment of gastrointestinal disturbances [4]. Many types of diarrheal illness with many different causes disrupt intestinal function. The ability of Probiotics to decrease the incidence or duration of certain diarrheal illnesses is the most substantiated health effect of probiotics. Lactobacillus is safe and effective as a treatment for children with acute infectious diarrhea. Probiotics have also been shown to decrease traveler's diarrhea and recurring colitis due to Clostridium difficile. Consumption of high levels ( 1010 per day) of certain strain of probiotic may shorten the duration or decrease the incidence of certain diarrheal illnesses [25]. A significant effect was observed in a study carried out with patients who presented diarrhea caused by antibiotics, in which intake of a probiotic drink containing $L$. casei, $L$. bulgaricuse and S. thermophilus reduced the incidence of diarrhea [4].

Diarrhea is frequent in the critically ill, especially in cases with sepsis and hypoalbuminaemia and during treatment with enteral nutrition. The standard treatment consists of liberal rehydration, replacement of electrolyte loss, use of anti-diarrhea remedies, and continuation with enteral nutrition. The benefit of enteral supplementation with soluble fiber, probiotics, or prebiotics is not clear [39].

In the treatment of rotavirus diarrhea, Lactobacillus is reported really effective. The best documented probiotic effect is shortened duration of rotavirus diarrhea using Lactobacillus. It has been given proof in several studies around the world by some researchers. One of types of diarrhea is traveler's diarrhea (TD) which affects the healthy travelers not only in developing countries but also in Europe [40]. Probiotics have beneficial effects in preventing some forms of traveler's diarrhea (TD). Antibiotic therapy causes mild and severe outbreaks of diarrhea. The normal microflora may be suppressed during the microbial therapy and resulting with filling with pathogenic strains. The changes of microflora may also encourage the resistant strains at least Clostridium difficile which is the reason of antibiotic associated diarrhea (AAD). Probiotics which are able to restore and replace the normal flora should be used [38]. 


\section{Veterinary importance of probiotics}

In animal nutrition microorganisms used as probiotics was linked with a proven efficacy on the gut microflora. Administration of probiotic strains separately and in combination was significantly improved feed intake, feed conversion rate, daily weight gain and total body weight in chicken, pig, sheep, goat, cattle and equine [41].

The probitics used as growth promoters permit to improve animal performance. Probiotics have been incorporated through diets, with the objective to keep intestinal microbiota balance of animals, preventing digestive tract diseases, improving feed digestibility, leading to a greater use of nutrients and improving animal performance [4]. Probiotics were stabilizers of ruminal $\mathrm{pH}$ and lactate, increased the absorption of some nutrients and displayed a growth-promoting effect that was comparable to avilamycin treatment [42].

Probiotic supplements are now widely used in quality animal production and accepted as agents that can bring significant health benefits such as: enhancement of the immune system; antimicrobial effects inhibiting intestinal and food poisoning pathogens; improvement of gut functions by normalizing microflora balance, reducing constipation and improving intestinal mobility; improved nutrition through the enhanced breakdown of vitamins, minerals and amino acids and their absorption through the intestinal walls; cleansing of the bloodstream by making it free of toxins and prevention of infection by harmful bacteria [16].

\section{Safety aspects of probiotics}

Most of the micro-organisms used as a probiotics in animal are safe, although some have problems particularly the enterococci, which may harbor transmissible antibiotic resistance determinants and Bacillus cereus group that are known to produce enterotoxins and an emetic toxin [43]. Particular attention for safety assessment is focused on the presence of transmissible antibiotic resistance markers and the potential for production of harmful metabolites. Thus the appealing properties of probiotics include the ability to reduce antibiotic use, the apparently high index of safety and the public's positive perception about natural or alternative therapies [44]. The main currently used tests for the study of probiotic strains are resistance to gastric acidity and bile acid, adhesion to gut epithelial tissue, antimicrobial activity against potentially pathogenic bacteria and ability to modulate immune responses [6].

Some species of lactobacilli and bifidobacteria are normal residents of or common transients through the human digestive system and as such do not display infectivity or toxicity. Traditional lactic acid bacteria (LAB), long associated with food fermentation, are generally considered safe for oral consumption as part of foods and supplements for the generally healthy population and at levels traditionally used. Regulations for dietary supplements are nonexistent in many countries, or much less strict than those that apply for prescription drugs [8]. There is concern over the use in foods of probiotic bacteria that contain specific drug resistance genes. Bacteria, which contain transmissible drug resistance genes, should not be used in foods [36]. To formalize and underwrite this concept, a system for a pre-market safety assessment was proposed that leads to a 'Qualified Presumption of Safety (QPS)' in the European Community. In summary, a safety assessment of selected groups of micro-organisms from a defined taxonomic group (e.g. genus or group of related species) can be made on the basis of four pillars of information (identity, body of knowledge, possible pathogenicity and end use) [5].

\section{Bacterial probiotics limitations}

There are numerous advantages and health benefits associated with probiotics or probiotic food products, there are risks associated with probiotic therapy. These risks are mainly concerned with respect to safety in vulnerable target groups such as immune compromised individuals (pregnant women, babies and the elderly) or critically ill or hospitalized patients [40]. Probitics can interact with commensal bacteria and can also have a direct impact on the host. Understanding these interactions is one of the key challenges for future research. Other key challenges are to understand their mechanisms of action, to elucidate more specifically which probiotic strains can offer which health benefits and to define the intake levels needed to achieve those effects [5].

Clinical and mechanistic studies are urgently required to better understand the interface between the microbes, host cells, mucus and immune defenses, and to create efficacious interventions. Such studies should include molecular examination of the intestinal (not only fecal) flora and long-term (5-10 years) effects of probiotic microorganisms [36]. Some have problems particularly the enterococci, which may harbor transmissible antibiotic resistance determinants and Bacillus cereus group that are known to produce enterotoxins and an emetic toxin [43]. Theoretically probiotics may responsible for four types of side-effects systemic infections, deleterious metabolic activities, excessive immune stimulation in susceptible individuals and Gene transfer [6]. Regulations for dietary supplements are nonexistent in many countries, or much less strict than those that apply for prescription drugs. Currently, the Food and Drug Administration (FDA) in the United States has not approved any claims for probiotics that relate probiotics to a reduction in the risk of disease [8]. The regulatory status of probiotics as a component in food is currently not established on an international basis. In only a few countries, regulatory procedures are in place or sufficiently developed to enable probiotic products to be allowed to describe specific health benefits [36]. About the use of probiotics persist because there are no conclusive studies on specific strains to be used, their formulations, and dose administration [45].

Factors affecting viability during storage such as temperature, moisture, and air should also be taken into consideration. Oxygen toxicity is another major problem in the survival of probiotic bacteria in dairy foods. High levels of oxygen in the product are 
detrimental to the viability of these anaerobic bacteria [46]. Retention of viability of the probiotic bacteria presents a major marketing and technological challenge for application of probiotic cultures in functional foods [47]. Many active cultures die during manufacturing, storage or transport of the finished product and also during the passage to the intestine [48]. Thus, the majority die even before the consumer receives any of the health benefits [49].

\section{Conclusion and Recommendations}

Probiotic supplements are now widely used in quality animal production and accepted as agents that can bring important health benefits such as antimicrobial effects inhibiting intestinal and food poisoning pathogens, improvement of gut functions by normalizing microflora balance, reducing constipation and improving intestinal mobility. Also it improves nutrition through the enhanced breakdown of vitamins, minerals and amino acids and their absorption through the intestinal walls and has role in the management of diarrhea. In addition to this, there is emerging evidence to indicate that probiotics can be taken by healthy people to prevent certain diseases and modulate their immunity. Probiotics have demonstrated an ability to prevent and treat some infections, particularly GI tract. Effective use of probiotics has the potential to decrease patients' exposure to antimicrobials. Available probiotics appear safe but should probably be avoided in patients at high risk for septicemia.

Therefore, based on the above conclusion the following recommendations are forwarded:

a) The probiotic microorganism should be able to confer defined health benefits on the host.

b) The regulatory status of probiotics as a component in food should be established on an international level.

c) Further advanced studies on probiotics as drugs should be enhanced.

d) Probiotics shouldn't be containing virulence, resistance genes or antibiotics resistance genes.

\section{References}

1. Eczema C (2013) Probiotics in animal production: A review. J Vet Med Anim Healt 5: 308-16.

2. Jim Q (2011) Direct-Fed Microbials (Probiotics) in Calf Diets. Bov Allia Mngmt Annutr 5: 1-4.

3. Chiquette J (2009) The Role of Probiotics in Promoting Dairy Production. Dair WCDS Adv Dair Techno 21:143-157.

4. Boaventura C, Rafael A, Ana U, Jacques N, Luis G (2012) The Benefits of Probiotics in Human and Animal Nutrition. NAB Clinical Gastroenterology. In Tech, Europe $75-100$.

5. Binns N (2013) ILSI Europe Concise Monograph Series Probiotics. Prebio Gut Microbi 2:1-31.

6. Musa HH, Wu SL, Zhu CH, Seri HI, Zhu G (2009) The Potential Benefits of Probiotics in Animal Production and Health. J Anim Vet Adv 8: $313-21$.

7. Sullivan A, Nord C (2005) Probiotics and gastrointestinal diseases. J Intern Med 257: 78-92.

8. Guarner F, Aamir G, Khan JG, Ram E, Alfred G, et al. (2008) Probiotics and probiotics Worl. Gastroentero. Organisa. Prac. Guideli 2:1-22.

9. Veizaj-Delia E, Piub T, Lekajc P, Tafaj M (2010) Using combined probiotic to improve growth performance of weaned piglets on extensive on farm conditions. Livest Scie 134: 249-51.

10. Boyington JE, Schoster B, Martin KR, Shreffler J, Callahan LF (2009) Perceptions of individual and community environmental influences on fruit and vegetable intake, North Carolina, 2004. Prev Chronic Dis 6: A04.

11. Kruis WP, Fric J, Pokrotnieks M, Lukas B, Fixa M, et al. (2004) Maintaining remission of ulcerative colitis with the probiotic E. coli Nissle 1917 is as effective as with standard mesalazine. Gut 53: 1617-23.

12. Ozyurt H, Otles S (2014) Properties of Probiotics and Encapsulate Probiotics in Food. Acta Sci Pol Techno Alimen 13: 413-24.

13. Henker J, Laass M, Blokhin BM, Bolbot YK, et al. (2007) The probiotic Escherichia coli strain Nissle 1917 (EcN) stops acute diarrhea in infants and toddlers. Eur JPediatr 166: 311-8.

14. Kruis W, Schutz E, Fric P, Fixa B, Judmaier G, et al. (2004) Double-blind comparison of an oral Escherichia coli preparation and mesalazine in maintaining remission of ulcerative colitis. Alim Pharm Ther 11: 853-8.

15. Tuomola E, Ross C, Martin P, Erika I, Seppo S (2001) Quality assurance criteria for probiotic bacteria. Am J Clin Nutr 73: 393-8.

16. Amiya K, Rautray R, Patra C, Sardar KK, Sahoo G (2011) Potential of Probiotics in Livestock Production. Eamr 1: 20-8.

17. Parvez S, Malik S, Ah-Kang H (2006) Probiotics and their fermented food products are beneficial for health. J Appl Microbiol 100: 1171-85.

18. Krehbiel R, Rust S, Zhang G, Gilliland E (2003) Bacterial direct-fed microbials in ruminant diets and its performance response and mode of action. J Anim Sci 81: 120-33.

19. Mercenier A, Pavan S, Pot B (2002) Probiotics as biotherapeutic agents. Present knowledge and future prospects. Curr Pharm Des 8: 99-110.

20. Phumkhachorn P, Rattanachai P (2010) Lactic acid bacteria their antimicrobial compounds and their uses in food production. Ann Bio Resear 1: 218-28.

21. Momose Y, Hirayama K, Itoh K (2008) Competition for proline between indigenous Escherichia coli and E. coli O157:H7 in gnotobiotic mice associated with infant intestinal microbiota and its contribution to the colonization resistance against E. coli O157:H7. Antonievan Leeuwenhoek 94: 165-71.

22. Ezendam J, Van Loveren H (2006) Probiotics: immunomodulation and evaluation of safety and efficacy. Nutr Rev 64: 1-14.

23. Penna F, Peret L, Vieira L, Nicoli J (2008) Probiotics and mucosal barrier in children. Curr Opin in Clin Nutr Metabol Car 11: 640-44.

24. Martins F, Dalmasso G, Arantes M, Doye A, Lemichez E, et al. (2010) Interaction of Saccharomyces boulardii with Salmonella enteric serovar Typhimurium protects mice and modifies T84 cell response to the infection. Plos One 5: 1932-2203.

25. Shinde PB (2012) Proobiotic: An Overview For Selction And Evaluation. Intern J Pharm Pharmaceu scie 4: 14-50.

26. Martins F, Elian S, Vieira A, Tiago F, Martins A, et al. (2011) Oral treatment with Saccharomyces cerevisiae strain UFMG 905 modulates immune responses and interferes with signal pathways involved in the activation of inflammation in a murine model of typhoid fever. Intern J Med Microbio 301: 359-64.

27. Boris AS (2015) Probiotics and Functional Foods. Encyclopedia of life support system (EOLSS). 
28. Knut JH (2001) Probiotic bacteria in fermented foods: product characteristics starter organisms. Am J Clin Nutr 73: 374-79.

29. Fuller R (2004) What is a probiotic? Bio 51: 232.

30. Danut G, Mocanu G, Rotaru A, Vasile E, Botez D, et al. (2009) Studies on the production of probiotic dairy products based on milk and medicinal plant extracts. J Agroalim Proces Techno 15: 234-38.

31. Aattour N, Bouras M, Tome D, Marcos A, Lemonnier D (2002) Oral ingestion of Lactic acid bacteria by rats increases lymphocyte proliferation and interferon production. Br J 87: 367-73.

32. Nicolae C, Dan Drinceanu L, Stef I, Calin Julean M (2010) Probiotics Identification and Ways of Action. Inno Romanian F Biotechno 6: 1-11.

33. Amel R, Zouhaier B, Mohammad R, Beatriz M, Ana R, Mohamed M, Nelson P (2014) Assessment of potential probiotic properties and multiple bacteriocin encoding- genes of the technological performing strain Enterococcus faecium. MMRA. F Contr 37: 343-50.

34. Shanahan F (2000) Probiotics and inflammatory bowel disease: Is there a scientific rationale? Inf Bow Dis 6: 107-15.

35. Gionchetti P, Rizzello F, Venturi A, Brigidi P, Matteuzzi D, et al. (2000) Oral bacteriotherapy as maintenance treatment in patients with chronic pouchitis a double-blind, placebo-controlled trial. Gastroent 119: 305-09.

36. Food and Agricultural organization (FAO) (2001) Expert Consultation on Evaluation of Health and Nutritional Properties of Probiotics in Food including Powder Milk with Live Lactic Acid Bacteria. 1-50.

37. Vahedi H, Ansari R, Nasseri M, Jafari E (2010) Irritable Bowel Syndrome: A Review Article. Mid E J Dgtv Dis 2: $2008-5249$.

38. Hatice Y (2007) Isolation, Characterization, Determination of Probiotic Properties of Lactic Acid Bacteria From Human Milk, A Thesis Submitted to The Graduate School of Engineering and Sciences of Izmir Institute of Technology In Partial Fulfillment of The Requirements For The Degree of Master of Science in Food Engineering. 1-69.

39. Wiesen P, Gossum A, Preiser J (2006) Diarrhea in the critically ill. Curr Opin Crit Car 12: 149-54.

40. Mercenier A, Jankovic I, Sybesma W, Phothirath P, Ananta E (2010) Application of probiotics in food products-challenges and new approaches. Curr Opin Biotechnol 21: 175-81

41. Samli H, Senkoylu N, Koc F, Kanter M, Agma A (2007) Effects of Enterococcus faecium and dried whey on broiler performance, gut histomorphology and intestinal microbiota. Arch Anim Nutr 61: 42-9.

42. Mountzouris K, Tsirtsikos E, Kalamara S, Nitsch G, Schatzmayr K (2007) Evaluation of the Efficacy of a Probiotic Containing Lactobacillus, Bifidobacterium, Enterococcus and Pediococcus Strains in Promoting Broiler Performance and Modulating Cecal Microflora Composition and Metabolic Activities. Poult Sci 86: 309-17.

43. Arturo A, Maria M, Maria A (2006) Probiotics for animal nutrition in the European Union. Regulation and safety assessment. Regul Toxic Pharm 45: 91-5.

44. Strompfova V, Marcinakova M, Simonva B, Bogovic M, Laukova A (2006) Application of potential probiotic Lactobacillus fermentum AD1 strain in healthy dogs. Anaerobe 12: 75-9.

45. Tahir H (2013) Probiotics and controversies. J Microbio Antimicrobio 5: 1.

46. Thantsha C, Mamvura I, Booyens J (2012) Probiotics What They Are, Their Benefits and Challenges. Ne Adv Bas Clin Gastroentero 5: $521-23$.

47. Desmond C, Stanton C, Fitzgerald GF, Collins K, Ross RP (2002) Environmental adaptation of probiotic lactobacilli towards improvement of performance during spray drying. Inter Dair J 12: 183-90.

48. Park J, Chang H (2000) Microencapsulation of microbial cells. Biotechno Adv 18: 303-19.

49. Siuta-Cruce P, Goulet J (2001) Improving probiotic microorganisms in food systems. F Techno 55: 36-42.

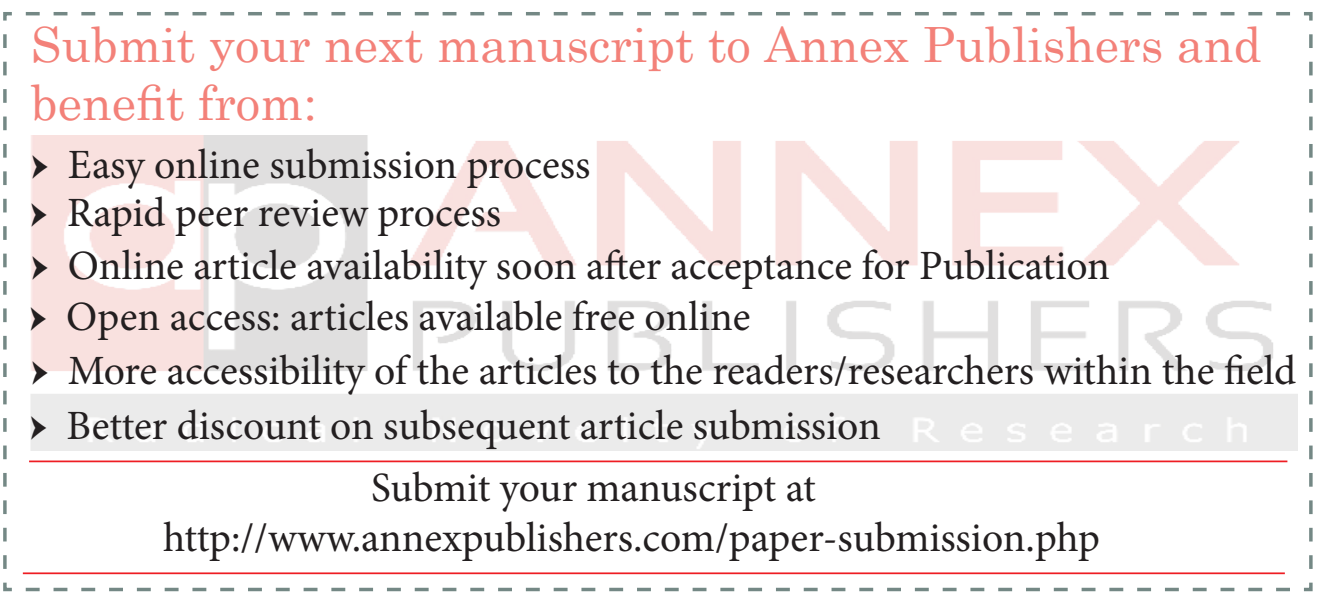

